\title{
Multi-objective Genetic Algorithms for Pipe Arrangement Design
}

\author{
Satoshi Ikehira \\ Dept. of Maritime Engineering \\ Graduate school of Engineering \\ Kyushu University \\ Fukuoka, Japan \\ ikehira@system.nams.kyushu-u.ac.jp
}

\author{
Hajime Kimura \\ Dept. of Marine Systems Engineering \\ Faculty of Engineering \\ Kyushu University \\ Fukuoka, Japan \\ kimura@nams.kyushu-u.ac.jp
}

\begin{abstract}
This paper presents an automatic design method for piping arrangement. A pipe arrangement design problem is proposed for a space in which many pipes and objects co-exist. This problem includes large-scale numerical optimization and combinatorial optimization problems, as well as two criteria. For these reasons, it is difficult to optimize the problem using usual optimization techniques such as Random Search. Therefore, multi-objective genetic algorithms suitable for this problem are developed. The proposed method for optimizing a pipe arrangement efficiently is demonstrated through several experiments.
\end{abstract}

\section{Categories and Subject Descriptors}

Real World Applications

\section{General Terms}

Algorithms, Design

\section{Keywords}

pipe arrangement, multi-objective optimization, genetic algorithm

\section{INTRODUCTION}

Recently, the shipbuilding industry has been able to integrate design and manufacturing systems due to advances in data processing technology. This integration promotes automation and reduces labor requirements, which have become necessary due to the lack of skilled workers. The design, analysis, and production of a given system can all be completed using three-dimensional data by employing CAD (Computer-Aided Design) software. It is quite difficult to visualize a complex pipe arrangement using a two-dimensional blueprint. For this reason, pipe arrangement designs have been generated using trial and error methods. Even now, a large part of a design relies upon the designer's experience, because the automation of pipe arrangements has not yet been achieved. A reason for this may be the underreporting of research on the automatic design of pipe arrangements.

This paper formulates a pipe arrangement design problem where many pipes co-exist in a close space and some obstacles must be avoided. This problem includes large-scale numerical optimization and combinatorial optimization problems, and has two criteria. Therefore, it is difficult to optimize the objective

Copyright is held by the author/owner(s).

GECCO'06, July 8-12, 2006, Seattle, Washington, USA.

ACM 1-59593-186-4/06/0007. using usual optimization techniques such as Random Search.

Therefore, multi-objective genetic algorithms suitable for this problem are proposed. It is demonstrated that the proposed method can optimize a pipe arrangement efficiently, and some remarks are provided on applying this method to a practical pipe arrangement design.

\section{FORMURATING PIPE ARRANGEMENT DESIGN PROBLEM}

\subsection{Generating Piping Route}

In this paper, piping route is automatically designed when both origin and endpoint coordinates and the directions in which the pipes expand (direction vectors) are provided.

There is an infinite number of piping routes that could be used to connect a given origin and endpoint. Therefore, a limit is imposed on the degrees-of-freedom for the route. A piping route is designed that essentially specifies the bending points (nodes). First, a route is designated as pattern-1, pattern-2, or pattern-3. Next, a shape is set according to the number of nodes and the relative position between the origin and the end-point, as shown in Figs. 1, 2 and 3. A pipe is characterized by both a pattern of generation and three numerical parameters that describe the length of the straight parts.

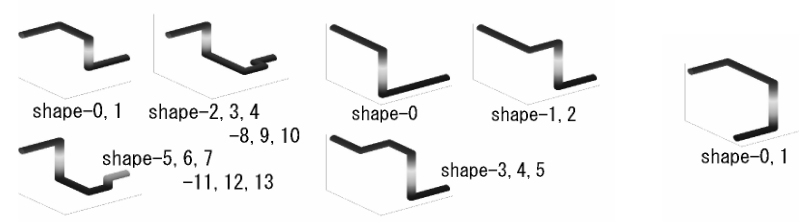

Figure 1: pattern-1 Figure 2: pattern-2 Figure 3: pattern3.

\subsection{The cost functions}

\subsubsection{The cost function for materials cost}

The cost function for materials cost is defined as the total sum of the length of pipes.

\subsubsection{The cost functions for obstacle}

The evaluation values are worse when a pipe passes through the center of an obstacle and the length of the intersection is long.

$$
f_{-} \text {obstacle }=\sum_{l=1}^{n_{o}} \sum_{k=1}^{n_{p}}\left(b_{k l}-\bar{a}_{k l}+A_{l}\right)
$$


Here, $k$ and $l$ are the index of each obstacle and pipe, respectively. $n_{o}$ and $n_{p}$ are the number of obstacles and pipes, respectively. $b_{k l}^{o}$ is the length of the intersection when a pipe $k$ intersects an obstacle $l . \bar{a}_{k l}$ is the averaged length between the center of the gravity of the obstacle $l$ and the part divided by each node of the pipe $k . A_{l}$ is the length between the center of the gravity and the top of the obstacle $l$.

\section{DESIGNING MULTI-OBJECTIVE GENETIC ALGORITHMS}

\subsection{Representation and Crossover}

A combination of both a pattern of generation and numerical parameters are used as the representation and is called generation gene.

A Crossover with Two Gene (XTG) is proposed. The XTG generates one child from two parents using a gene named the obstacle gene, which contains the obstacle interference information, as shown in Fig. 4.

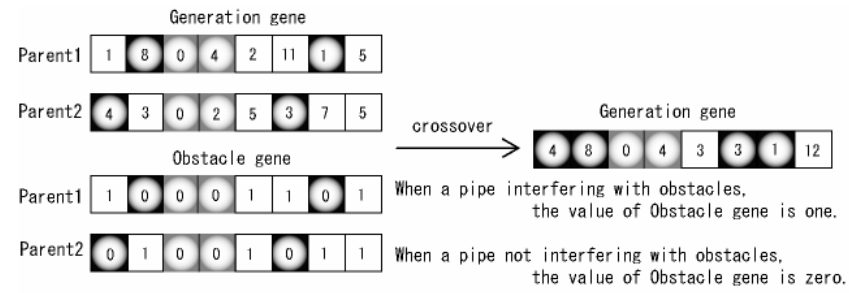

Figure 4: An example of the Crossover with Two Gene (XTG).

\subsection{Generation-Alternation Model}

A generation-alternation model based on the Pareto optimal selection strategy, as shown in Fig. 5(Yoshida [2]), is employed.

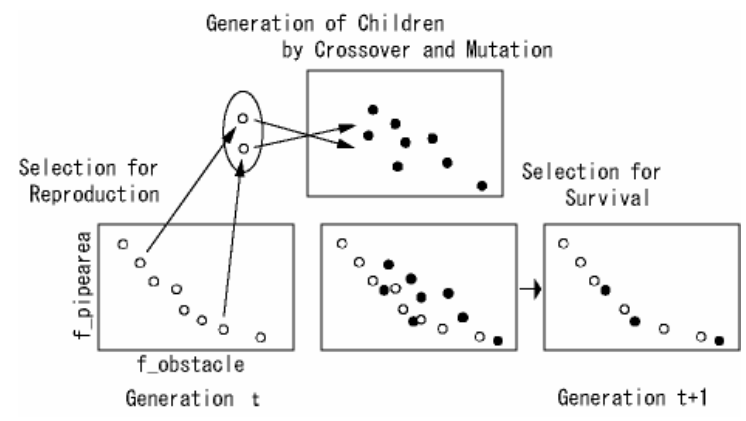

Figure 5: Generation-alternation model.

\subsection{Modification Operator to Avoid Contact with pipes}

In this paper, a solution candidate is regarded as infeasible when pipes interfere with each other. When initial solution candidates are generated and new solution candidates are created, the probability of pipes avoiding interference with each other rapidly decreases as the number of pipes increases. To overcome this problem, a modification operator is proposed that makes infeasible solutions candidates feasible, which changes the properties of the original solution candidates as little as possible.

\subsection{Result}

To confirm the effectiveness of the proposed method, it was applied to a 15 pipes arrangement problem.

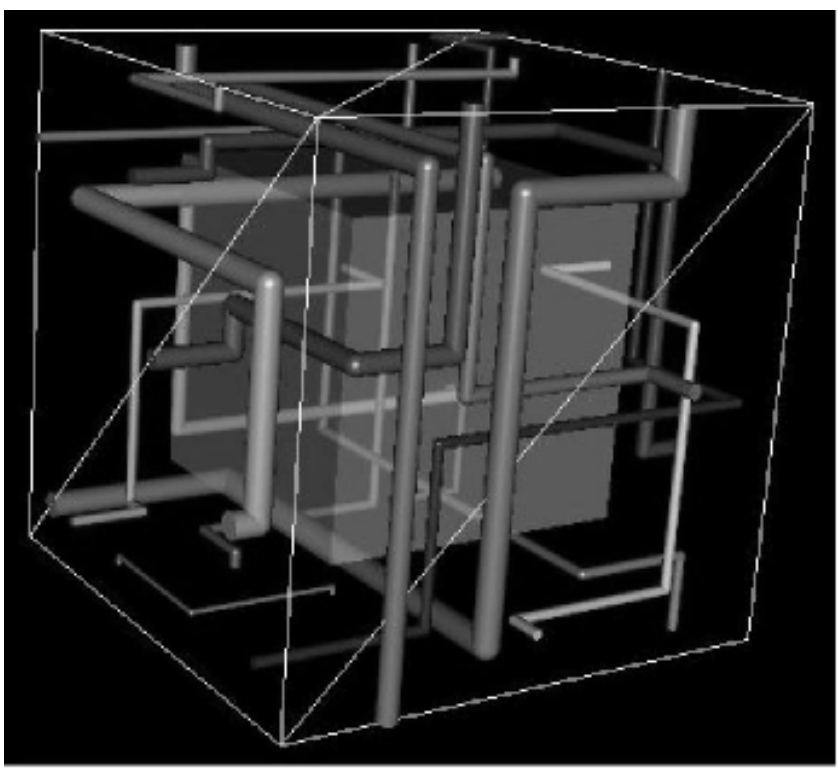

Figure 6: Result of 15 pipes arrangement.

\section{CONCLUSIONS}

In this paper, a design problem for pipe arrangement in a small space where many pipes and obstacles co-exist was formulated, and a multi-objective Genetic Algorithm suitable for this problem was developed.

In the future, the work efficiency for installing a pipe and doing maintenance should be considered, and the design should be integrated with the production process. Moreover, fluid resistance, vibration or strength of structure should be considered as a cost function. Because the search time increases as the number of pipes increases, improvements to the proposed algorithms should be continued.

\section{ACKNOWLEDGMENTS}

The authors thank Mr. Eisuke Ikezaki at Fukuoka Shipbuilding Co., Ltd for introducing the practical problem on pipe arrangement design kindly.

\section{REFERENCES}

[1] S.Kobayashi, K.Yoshida, and M.Yamamura. Generating pareto optimal decision trees by genetic algorithms. The Japanese Society for Artificial Intelligence, 11(5):778--785, 1996.

[2] I.Ono. Genetic Algorithms for Optimization Taking Account of Characteristics Preservation. Ph.D. dissertation, Tokyo Institute of Technology, 1997. 